\title{
Ab-Initio Study on Structural and Magnetic Properties of Fe-Doped MnCoGe
}

\author{
Kaho Nagano, Yuri Okubo, Yoshifuru Mitsui, Keiichi Koyama and Shinpei Fujii* \\ Graduate School of Science \& Engineering Kagoshima University, Kagoshima 890-0065, Japan
}

First-principles calculations were performed to investigate the effect of Fe substitution on the structural transformation of (Mn,Fe)CoGe and $\mathrm{Mn}(\mathrm{Co}, \mathrm{Fe}) \mathrm{Ge}$. The activation energy (barrier) between orthorhombic and hexagonal structures was estimated from the total energy of each of several virtual structures between them.

Fe substitution reduces the activation energy, and movements of both of Co and $\mathrm{Mn}$ are closely related to the reduction. Moreover, the calculation result for the Fe substitution at the sites of both Mn and Co indicates that Fe atoms randomly occupy Mn and Co sites. [doi:10.2320/matertrans.MT-MN2019013]

(Received December 24, 2019; Accepted February 13, 2020; Published March 23, 2020)

Keywords: Fe-doped, MnCoGe, Ni $i_{2} I n$-type, TiNiSi-type, activation energy

\section{Introduction}

In the MnCoGe alloy, the structural transformation from a $\mathrm{Ni}_{2}$ In-type hexagonal structure (space group $P 6_{3} / m m c$, No. 194) to a TiNiSi-type orthorhombic structure (space group Pnma, No. 62) at $375-620 \mathrm{~K}\left(T_{\mathrm{m}}\right)$ is followed by the magnetic transformation from a paramagnetic state to a ferromagnetic state at $327-355 \mathrm{~K}\left(T_{\mathrm{c}}\right){ }^{1-4)}$ The two crystal structures were shown in Fig. 1. When the crystal structure changes from a hexagonal phase to an orthorhombic one, a large volume change and a large increase in magnetic moment occur. ${ }^{1,2,5)}$ If the $T_{\mathrm{m}}$ is lowered below the $T_{\mathrm{c}}$, two transitions, i.e., structural and magnetic, can occur simultaneously and can cause an improvement of the magnetocaloric effect. Accordingly, several experimental studies have been conducted such as introducing a vacancy, ${ }^{6}$ ) and fourth elements ${ }^{4,7-9)}$ in MnCoGe. Recently, several experimental studies on $(\mathrm{Mn}, \mathrm{Fe}) \mathrm{CoGe}^{3,13-15)}$ and $\mathrm{Mn}(\mathrm{Co}, \mathrm{Fe}) \mathrm{Ge}^{3,10-12)}$ have shown that the Fe substitution produces a decrease in the $T_{\mathrm{m}}$ below the $T_{\mathrm{c}}$. Thus, these materials can be used as a magnetic functional material in a magnetic-field-driven actuator or a magnetic refrigeration material. ${ }^{10,16)}$

In this study, we have investigated the structural transformation from a hexagonal ferromagnetic structure to an orthorhombic ferromagnetic one. ${ }^{1,12)}$ We have performed first-principles calculations for the $(\mathrm{Co}, \mathrm{Fe}) \mathrm{MnGe}$ and $\mathrm{Co}(\mathrm{Mn}, \mathrm{Fe}) \mathrm{Ge}$ systems. We will discuss the structural transformation from the point of view of an activation energy (barrier) between two structures, which is estimated from first-principles calculations.

\section{Approach}

We used a $2 \times 2 \times 1(2 \times 2 \times 2)$ supercell to simulate a one-quarter (one-eighth) substituted hexagonal system with the lattice vectors $\boldsymbol{a}_{\mathrm{h}}, \boldsymbol{b}_{\mathrm{h}}$, and $\boldsymbol{c}_{\mathrm{h}}$. We used a $1 \times 2 \times 2$ $(2 \times 2 \times 2)$ supercell to simulate a one-quarter (one-eighth) substituted orthorhombic system with the lattice vectors $\boldsymbol{a}_{\mathrm{o}}$, $\boldsymbol{b}_{\mathrm{o}}$, and $\boldsymbol{c}_{\mathrm{o}}$. We optimized the lattice constants of the hexagonal and orthorhombic structures by minimizing the

*Corresponding author, E-mail: fujii@sci.kagoshima-u.ac.jp total energy. The atomic positions of the hexagonal and orthorhombic structures were optimized by minimizing the forces acting on the atoms. The results are listed in Table 1, where we only show the atomic positions in the orthorhombic MnCoGe. The first-principles calculations on an electronic structure were performed using the full-potential linearized augmented plane wave method. ${ }^{17)}$ The generalized gradient approximation developed by Perdew et al. ${ }^{18)}$ was used to determine the exchange-correlation potential. The plane wave cutoff was $\mathrm{RK}_{\max }=10.0$, where $\mathrm{R}$ is the smallest atomic sphere radius and $\mathrm{K}_{\max }$ is the magnitude of the largest $\mathrm{K}$ vector. The maximum $l$ value for the partial waves used inside the atomic spheres was 10. For the atomic sphere radius, the following values were used: 2.2 a.u. for the $3 \mathrm{~d}$ transition metals and 1.9 a.u. for Ge. To generate $\mathrm{k}$ points, $14 \times 14 \times 9$ and $9 \times 14 \times 7$ divisions of the Brillouin Zone were used for the hexagonal and orthorhombic structures, respectively.

\section{Results and Discussions}

\subsection{Preliminary results}

Figure 2 shows the total energy as a function of the volume for the hexagonal and orthorhombic phases in the paramagnetic (non-magnetic) and ferromagnetic states, for which the optimal lattice constants and atomic positions are listed in Table 1. This figure shows that a ferromagnetic orthorhombic phase is the most stable state. The values changes by $2.8 \%$ and $5.2 \%$ between the two structures in paramagnetic and ferromagnetic states, respectively. These values are close to the values reported previously for a paramagnetic state $\left(4.1 \%^{11)}\right)$ and for a ferromagnetic state $\left(5.5 \%^{19)}\right.$ and $\left.4.4 \%^{12)}\right)$.

\subsection{Activation energy}

We prepared other nine structures with the following lattice constants: $x_{\mathrm{j}}=x_{0}+\mathrm{j}^{*}\left(x_{10}-x_{0}\right) / 10 ; \mathrm{j}=0,1,2, \ldots 10 ; x_{0}=$ $\boldsymbol{a}_{\mathrm{o}}, \boldsymbol{b}_{\mathrm{o}}, \boldsymbol{c}_{\mathrm{o}} ; x_{10}=\boldsymbol{c}_{\mathrm{h}}, \boldsymbol{a}_{\mathrm{h}}, \boldsymbol{a}_{\mathrm{h}}+2 \boldsymbol{b}_{\mathrm{h}}$ to estimate the activation energy (barrier) between the two structures. For these nine structures, we optimized the atomic positions of the atoms in the cell. The total energies obtained for the 11 structures are shown in Fig. 3(a). The energy difference between each 
(a)

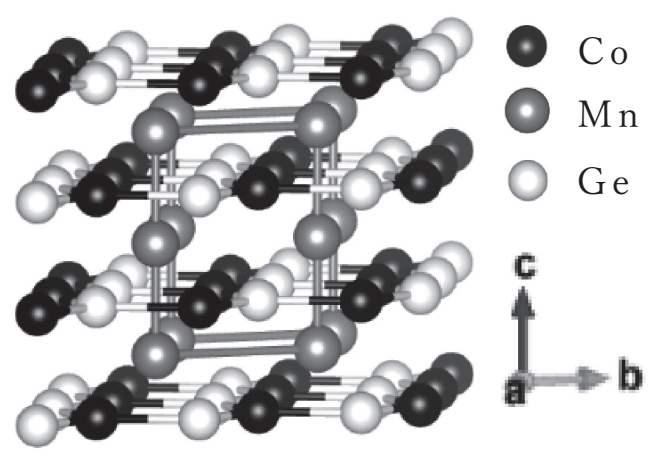

(b)

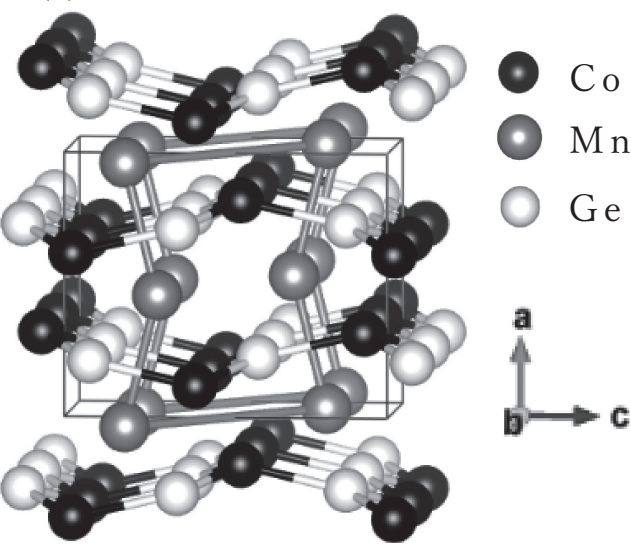

Fig. 1 Hexagonal (a) and orthorhombic (b) structures.

Table 1 Optimized lattice constants $(\AA)$ of $\left(\mathrm{Mn}_{1-\mathrm{x}} \mathrm{Fe}_{\mathrm{x}}\right)\left(\mathrm{Co}_{1-\mathrm{y}} \mathrm{Fe}_{\mathrm{y}}\right) \mathrm{Ge}$. Optimized atomic positions of orthorhombic $\mathrm{Mn}, \mathrm{Co}$, and $\mathrm{Ge}$ are listed at 3rd, 4th and 5th columns. Optimized atomic positions in other materials are not listed.

\begin{tabular}{|c|c|c|c|c|}
\hline $\mathrm{x}$ & $\mathrm{y}$ & $a$ & $b$ & $c$ \\
\hline \multicolumn{5}{|c|}{ Orthorhombic } \\
\hline \multirow[t]{4}{*}{0.00} & 0.00 & 5.813 & 3.782 & 7.108 \\
\hline & $\mathrm{Co}$ & 0.841 & 0.250 & 0.440 \\
\hline & $\mathrm{Mn}$ & 0.968 & 0.250 & 0.192 \\
\hline & $\mathrm{Ge}$ & 0.242 & 0.250 & 0.620 \\
\hline 0.25 & 0.00 & 5.800 & 3.773 & 7.092 \\
\hline 0.00 & 0.25 & 5.813 & 3.782 & 7.108 \\
\hline \multicolumn{5}{|c|}{ Hex a g o n a 1} \\
\hline 0.00 & 0.00 & 4.085 & 4.085 & 5.142 \\
\hline 0.25 & 0.00 & 4.089 & 4.089 & 5.100 \\
\hline 0.00 & 0.25 & 4.084 & 4.084 & 5.108 \\
\hline
\end{tabular}

structure and the orthorhombic one is maximum at a step 7 $(j=7)$. We regard this value as the activation energy from a orthorhombic phase to a hexagonal one. This figure shows that the activation energy decreases with $\mathrm{Fe}$ substitution and that the amount of change is larger in $\mathrm{Mn}\left(\mathrm{Co}_{0.75} \mathrm{Fe}_{0.25}\right) \mathrm{Ge}$ than in $\left(\mathrm{Mn}_{0.75} \mathrm{Fe}_{0.25}\right) \mathrm{CoGe}$.

The magnetization per formula unit is shown in Fig. 3(b). This figure shows the following aspects. The magnetization, $M$, decreases from step 0 (orthorhombic phase) to step 10 (hexagonal phase). The $M$ of $\mathrm{Mn}\left(\mathrm{Co}_{0.75} \mathrm{Fe}_{0.25}\right) \mathrm{Ge}$ is almost same as that of $\mathrm{MnCoGe}$, but the $M$ of $\left(\mathrm{Mn}_{0.75} \mathrm{Fe}_{0.25}\right) \mathrm{CoGe}$ is lower than that of MnCoGe. Larger magnetocaloric effects are expected for $\mathrm{Mn}(\mathrm{Co}, \mathrm{Fe}) \mathrm{Ge}$ than for $(\mathrm{Mn}, \mathrm{Fe}) \mathrm{CoGe}$ because the change in $M$ from orthorhombic to hexagonal phases is larger for $\mathrm{Mn}(\mathrm{Co}, \mathrm{Fe}) \mathrm{Ge}$ than for $(\mathrm{Mn}, \mathrm{Fe}) \mathrm{CoGe}$.

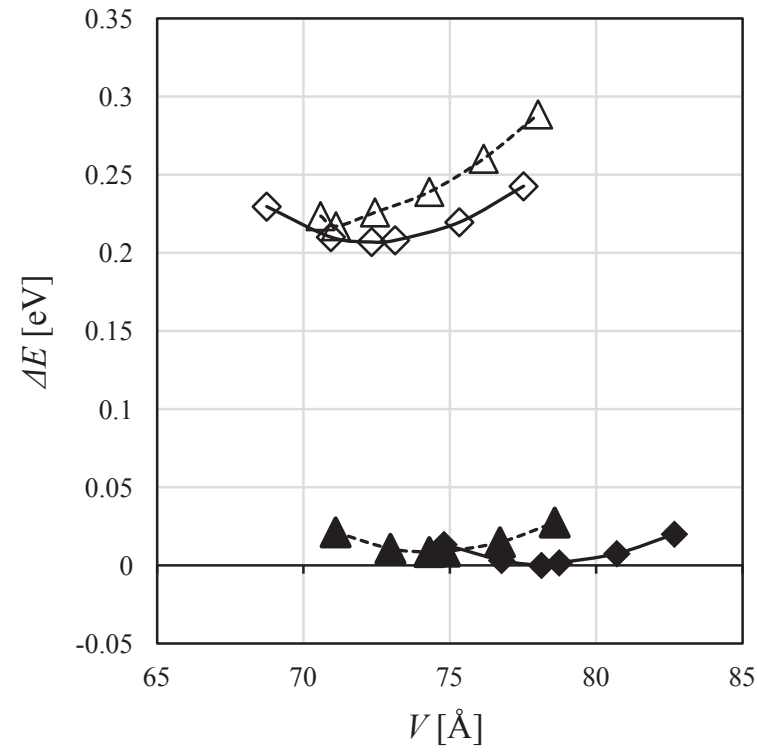

Fig. 2 Total energy of MnCoGe as a function of the volume par two formula units. The solid and dotted lines show the orthorhombic and hexagonal phases. White (black) triangle and diamond symbols show the paramagnetic (ferromagnetic) state.

We show the atomic arrangements of steps 0,8 , and 10 from the view of the bo axis in Fig. 4. This figure indicates that the movement of Co $(\mathrm{Mn})$ is remarkable from steps 10 to 8 (8 to 0$)$. To verify this, we estimate an amount of movement of $\mathrm{Mn}, \mathrm{Co}$, and $\mathrm{Ge}$ and the results are listed in Table 2. Here, we listed the first three atoms with the largest movement. The amount of movement of Ge is smaller than the amounts of movement of $\mathrm{Co}$ and $\mathrm{Mn}$, and hence, it is not shown. This table validates the arrangement shown in Fig. 4. However, the result for steps 0 to 6 shows that the amount of movement is almost the same in both Co and Mn. This is also observed in steps 6 to 10 . Thus, the activation energy is closely related to the movements of both $\mathrm{Co}$ and $\mathrm{Mn}$, and the contribution of the movement of $\mathrm{Ge}$ is small.

To investigate the relationship between $\mathrm{Fe}$ concentration and the stability of the structure, we have prepared an additional structure $\mathrm{Mn}\left(\mathrm{Co}_{0.625} \mathrm{Fe}_{0.375}\right) \mathrm{Ge}$, whose lattice constants and atomic positions are fixed to the corresponding values of hexagonal and orthorhombic MnCoGe. The result 

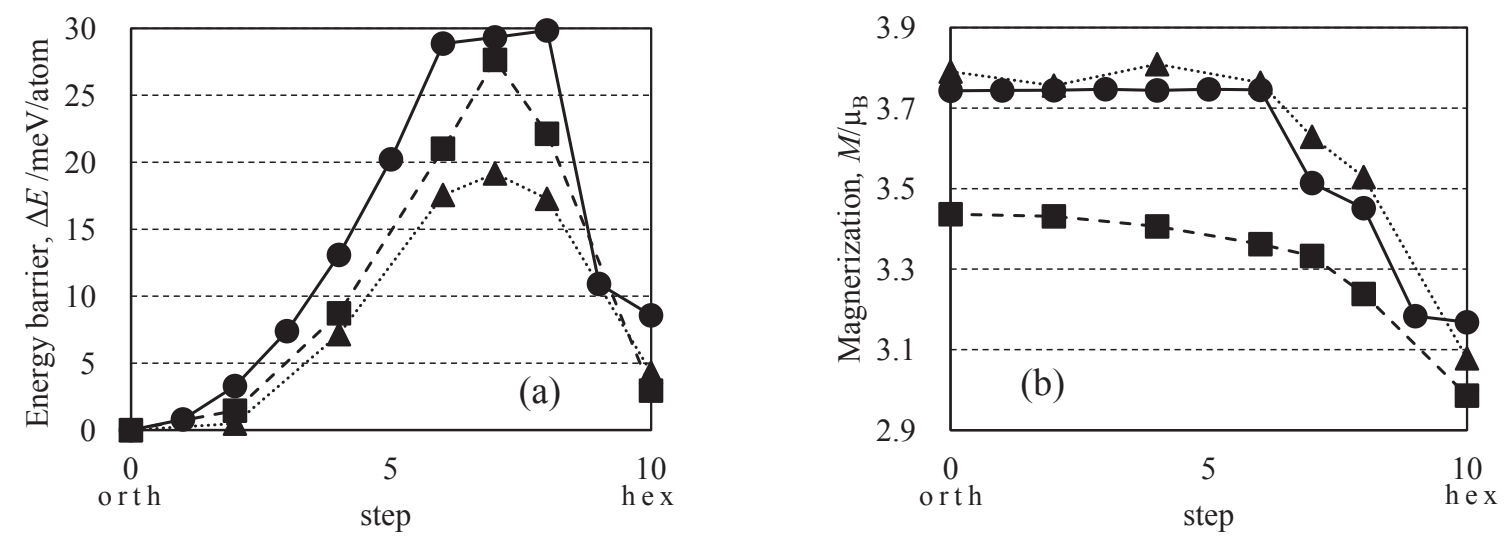

- $\mathrm{MnCoGe} \mathrm{Mn}_{0.75} \mathrm{Fe}_{0.25} \mathrm{CoGe} \boldsymbol{\Delta} \mathrm{MnCo}_{0.75} \mathrm{Fe}_{0.25} \mathrm{Ge}$

Fig. 3 Energy barrier (a) and magnetization change (b) during phase transition for the $\mathrm{MnCoGe}(\mathrm{circle}), \mathrm{Mn}_{0.75} \mathrm{Fe}_{0.25} \mathrm{CoGe}(\mathrm{square})$ and $\mathrm{MnCo}_{0.75} \mathrm{Fe}_{0.25} \mathrm{Ge}$ (triangle). The terms, "orth" and "hex", indicate orthorhombic and hexagonal structures, respectively.
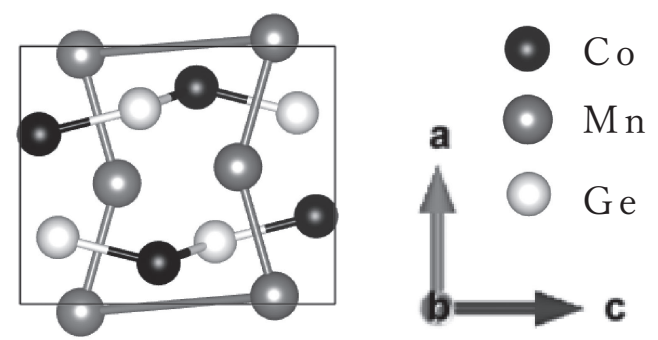

step0 (orthorhombic)

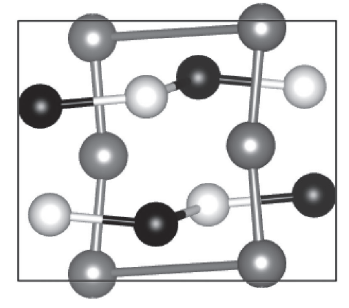

s te p 8

step 10 (hexagonal)

Fig. 4 Atomic arrangement from the view of the $\boldsymbol{b}_{\mathrm{o}}$ axis.

Table 2 Amount of atomic movement.

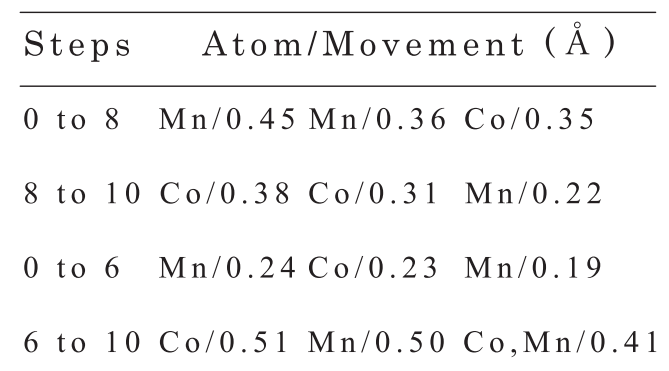

are shown in Fig. 5. The energy difference between the hexagonal and orthorhombic phases almost disappears in $\mathrm{Mn}\left(\mathrm{Co}_{0.625} \mathrm{Fe}_{0.375}\right) \mathrm{Ge}$. Our results indicate that, for $x \geq x_{c}$ $(=0.375)$, the hexagonal structure is more stable than the orthorhombic one. This value of $x_{c}$ is larger than those estimated from the experimental results such as $0.25^{10)}$ or $0.08 .^{11)}$ Thus, we conducted a trial calculation of

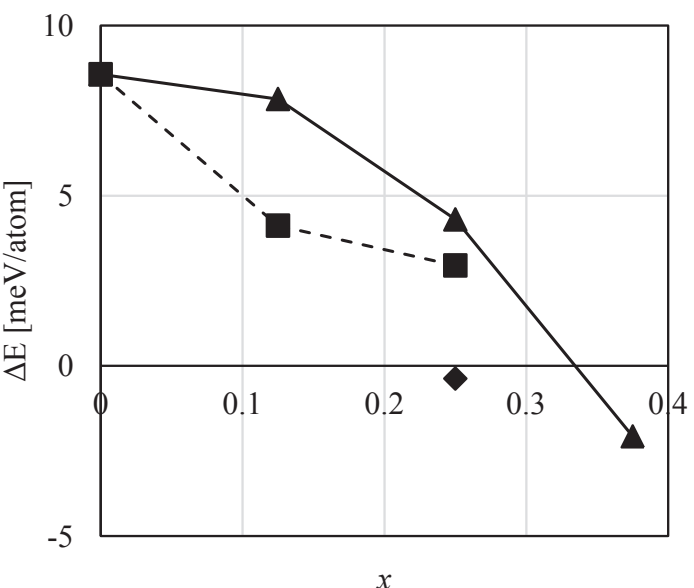

Fig. 5 Energy difference as a function of concentration of $\mathrm{Fe}$ in the $\mathrm{Mn}\left(\mathrm{Co}_{1-\mathrm{x}} \mathrm{Fe}_{\mathrm{x}}\right) \mathrm{Ge}$ (triangle), $\left(\mathrm{Mn}_{1-\mathrm{x}} \mathrm{Fe}_{\mathrm{x}}\right) \mathrm{CoGe}$ (square) and $\left(\mathrm{Mn}_{0.875} \mathrm{Fe}_{0.125}\right)\left(\mathrm{Co}_{0.875} \mathrm{Fe}_{0.125}\right) \mathrm{Ge}$ (diamond).

$\left(\mathrm{Mn}_{0.875} \mathrm{Fe}_{0.125}\right)\left(\mathrm{Co}_{0.875} \mathrm{Fe}_{0.125}\right) \mathrm{Ge}$, whose lattice constants and atomic positions are fixed to the corresponding values of hexagonal and orthorhombic $\mathrm{Mn}\left(\mathrm{Co}_{0.875} \mathrm{Fe}_{0.125}\right) \mathrm{Ge}^{20}{ }^{20}$ In this case, the hexagonal phase is lower in energy than the orthorhombic phase. This result indicates a random occupation of $\mathrm{Fe}$ at both $\mathrm{Mn}$ and Co sites.

\section{Summary}

We investigated the structural transformation from the point of view of the activation energy (barrier) between the hexagonal and orthorhombic structures, which is estimated from the first-principles calculations. We showed that the Fe substitution decreases the activation energy. We discussed the relationship between the activation energy and the atomic movement. We indicated the possibility that $\mathrm{Fe}$ atoms randomly occupy $\mathrm{Mn}$ and Co sites.

\section{Acknowledgments}

This research is supported by a Grant-in-Aid from Scientific Research, JP17K06840. 


\section{REFERENCES}

1) V. Johnson: Inorg. Chem. 14 (1975) 1117.

2) T. Kanomata, H. Ishigaki, T. Suzuki, H. Yoshida, S. Abe and T. Kaneko: J. Magn. Magn. Mater. 140-144 (1995) 131-132.

3) G.J. Li, E.K. Liu, H.G. Zhang, Y.J. Zhang, J.L. Chen, W.H. Wang, H.W. Zhang, G.H. Wu and S.Y. Yu: J. Magn. Magn. Mater. 332 (2013) 146.

4) D. Choudhury, T. Suzuki, Y. Tokura and Y. Taguchi: Sci. Rep. 4 (2015) 7544.

5) T. Kanomata, H. Ishigaki, K. Sato, M. Sato, T. Shinohara, F. Wagatsuma and T. Kaneko: J. Magn. Soc. Jap. 23 (1999) 418.

6) E.K. Liu, W. Zhu, L. Feng, J.L. Chen, W.H. Wang, G.H. Wu, H.Y. Liu, F.B. Meng, H.Z. Luo and Y.X. Li: EPL 91 (2010) 17003.

7) N.T. Trung, V. Biharie, L. Zhang, L. Caron, K.H.J. Buschow and E. Brück: Appl. Phys. Lett. 96 (2010) 162507.

8) N.T. Trung, L. Zhang, L. Caron, K.H.J. Buschow and E. Brück: Appl. Phys. Lett. 96 (2010) 172504.

9) T. Samanta, I. Dubenko, A. Quetz, S. Stadler and N. Ali: Appl. Phys. Lett. 101 (2012) 242405.

10) J. Zeng, Z. Wang, Z. Nie and Y. Wang: Intermetallics 52 (2014) 101.

11) K. Ozono, Y. Mitsui, M. Hiroi, R.Y. Umetsu, K. Takahashi, K. Matsubayashi, Y. Uwatoko and K. Koyama: Mater. Trans. 57 (2016)
316.

12) M. Onoue, R. Kobayashi, Y. Mitsui, M. Hiroi, K. Takahashi, A. Kondo, K. Kindo, Y. Uwatoko and K. Koyama: Mater. Trans. 59 (2018) 1645.

13) T. Samanta, I. Dubenko, A. Quetz, S. Stadler and N. Ali: J. Magn. Magn. Mater. 330 (2013) 88

14) Z. Wang, Z. Nie, J. Zeng, R. Su, Y. Zhang, D.E. Brown, Y. Ren and Y. Wang: J. Alloy. Compd. 577 (2013) 486.

15) T. Samanta, I. Dubenko, A. Quetz, J. Prestigiacomo, P.W. Adams, S. Stadler and N. Ali: Appl. Phys. Lett. 103 (2013) 042408.

16) V.K. Pecharsky and K.A. Gschneidner, Jr.: J. Magn. Magn. Mater. 200 (1999) 44.

17) P. Blaha, K. Schwarz, G.K.H. Madsen, D. Kvasnicka and J. Luitz: WIEN2k, An Augmented Plane Wave + Local Orbitals Program for Calculating Crystal Propaties, (Karlheinz Schwarz, Techn. Universitat Wien, Austria, 2001).

18) J.P. Perdew, K. Burke and M. Ernzerhof: Phys. Rev. Lett. 77 (1996) 3865.

19) K. Koyama, M. Sakai, T. Kanomata and K. Watanabe: Jpn. J. Appl. Phys. 43 (2004) 8036.

20) For this material, we do not show such data as optimized lattice constants and atomic positions in this paper. The data will appear in an upcoming paper. 